\title{
Hybrid Iterative Multiuser Detection for Channel Coded Space Division Multiple Access OFDM Systems
}

\author{
Ming Jiang, Student Member, IEEE, Soon Xin Ng, Member, IEEE, and Lajos Hanzo, Fellow, IEEE
}

\begin{abstract}
Space division multiple access (SDMA) aided orthogonal frequency division multiplexing (OFDM) systems assisted by efficient multiuser detection (MUD) techniques have recently attracted intensive research interests. The maximum likelihood detection (MLD) arrangement was found to attain the best performance, although this was achieved at the cost of a computational complexity, which increases exponentially both with the number of users and with the number of bits per symbol transmitted by higher order modulation schemes. By contrast, the minimum mean-square error (MMSE) SDMA-MUD exhibits a lower complexity at the cost of a performance loss. Forward error correction (FEC) schemes such as, for example, turbo trellis coded modulation (TTCM), may be efficiently combined with SDMA-OFDM systems for the sake of improving the achievable performance. Genetic algorithm (GA) based multiuser detection techniques have been shown to provide a good performance in MUD-aided code division multiple access (CDMA) systems. In this contribution, a GA-aided MMSE MUD is proposed for employment in a TTCMassisted SDMA-OFDM system, which is capable of achieving a similar performance to that attained by its optimum MLD-aided counterpart at a significantly lower complexity, especially at high user loads. Moreover, when the proposed biased $Q$-function based mutation (BQM) assisted iterative GA (IGA) MUD is employed, the GA-aided system's performance can be further improved, for example, by reducing the bit error ratio (BER) measured at $3 \mathrm{~dB}$ by about five orders of magnitude in comparison to the TTCMassisted MMSE-SDMA-OFDM benchmarker system, while still maintaining modest complexity.
\end{abstract}

Index Terms-Biased $Q$-function based mutation (BQM), genetic algorithm (GA), minimum mean-square error (MMSE), multiuser detection (MUD), orthogonal frequency division multiplexing (OFDM), space division multiple access (SDMA), turbo trellis coded modulation (TTCM).

\section{INTRODUCTION}

$\mathbf{S}$ PACE DIVISION multiple access (SDMA) based orthogonal frequency division multiplexing (OFDM) [1] communication invoking multiuser detection (MUD) techniques has recently attracted intensive research interests. In SDMA multi-input-multi-output (MIMO) systems, the transmitted signals of $L$ simultaneous uplink mobile users-each equipped with a single transmit antenna-are received by the

Manuscript received November 7, 2004; revised May 1, 2005. This work was supported in part by the Wireless Enablers 2.2 of the Core 3 Research Programme of the Virtual Centre of Excellence in Mobile and Personal Communications, Mobile VCE. The review of this paper was coordinated by Dr. M. Valenti.

The authors are with the School of Electrical and Computer Science, University of Southampton, Southampton SO17 1BJ, U.K. (e-mail: mj02r@ecs. soton.ac.uk; sxn@ecs.soton.ac.uk; lh@ecs.soton.ac.uk).

Digital Object Identifier 10.1109/TVT.2005.861187
$P$ different receiver antennas of the base station (BS). At the BS, the individual user's signals are separated with the aid of their unique, user-specific spatial signature constituted by their channel transfer functions or, equivalently, channel impulse responses (CIRs). A variety of MUD schemes, such as the least squares (LS) [1]-[3] and minimum mean-square error (MMSE) [1]-[5] detectors, or successive interference cancellation (SIC) [1]-[6], parallel interference cancellation (PIC) [1], [2], [6], [7] and maximum likelihood detection (MLD) [1], [2], [4], [5], [8] schemes may be invoked for the sake of separating the different users at the BS on a per-subcarrier basis. Among these schemes, the ML detection arrangement was found to give the best performance, although this was achieved at the cost of a dramatically increased computational complexity, especially in the context of a high number of users and higher order modulation schemes, such as 16QAM [6]. By contrast, MMSE combining exhibits the lowest complexity in this set of detectors, while suffering from a performance loss [1], [6]. Moreover, another branch of multiuser detection schemes referred to as sphere decoder (SD) [9]-[15] has also been proposed for multiuser systems, which is capable of achieving ML performance at a lower complexity. However, as far as we are concerned, most of the previously mentioned techniques were proposed for the systems where the number of users is less than or equal to the number of receivers.

Furthermore, the achievable performance can be significantly improved if forward error correction (FEC) schemes, such as, for example, turbo convolutional (TC) codes [16], are incorporated into the SDMA system. Various coded modulation (CM) schemes such as, for example, trellis coded modulation (TCM) [16], [17]; turbo TCM (TTCM) [16], [18], bit-interleaved coded modulation (BICM) [16], [19]; and iteratively decoded BICM (BICM-ID) [16], [20] have also attracted intensive research interests, since they are capable of achieving a substantial coding gain without bandwidth expansion. It was demonstrated in [21] that TTCM generally provides the best performance in the family of CM schemes in the specific context of the SDMAOFDM system investigated. Hence, in this paper, we will adopt TTCM as the FEC scheme for our SDMA-OFDM system.

Genetic algorithms (GAs) [22]-[26] have been applied to a number of problems, such as machine learning and modeling adaptive processes. Moreover, GA-based multiuser detection has been proposed by Juntti et al. [27] and Wang et al. [28], where the analysis was based on the additive white Gaussian noise (AWGN) channel in the absence of diversity techniques. The proposal by Ergün et al. [29] utilized GAs as the first stage of 
a multistage multiuser detector, in order to provide good initial guesses for the subsequent stages. Its employment in Rayleigh fading channels was considered by Yen et al. in [30], [31], and [31], [32] in diverse scenarios, both with and without the aid of diversity techniques, respectively.

However, most of the GA-aided transceiver research previously mentioned was conducted in the context of code division multiple access (CDMA) systems [31], [33]. Against this background, the novel contribution of this paper is that we apply new GAs in the context of novel multiuser OFDM rather than CDMA systems. Specifically, we propose a novel GA and MMSE-MUD assisted TTCM-aided SDMA-OFDM system which is capable of maintaining near-optimum performance in so-called overloaded systems, where the number of users is higher than the number of receiver antenna elements. Our simulation results show that the proposed GA assisted TTCM-SDMA-OFDM system is capable of achieving performance similar to that attained by its optimum but high-complexity maximum likelihood (ML) MUD assisted counterpart in overloaded scenarios, while imposing a significantly lower computational complexity, especially at high user loads. Furthermore, the performance of the proposed GA-aided system can be further improved if the proposed biased $Q$-function based mutation (BQM) aided iterative GA (IGA) MUD is employed. We will show that the BQMIGA aided system is capable of reducing the bit error ratio (BER) by up to five orders of magnitude in comparison to the TTCM-assisted MMSE-SDMA-OFDM benchmarker system. Moreover, the complexity of the proposed detection scheme is only moderately higher than that imposed by the linear MMSE MUD, and is substantially lower than that imposed by the optimum ML MUD.

The structure of this paper is as follows. The SDMA MIMO channel model is described in Section II-A and the overview of the GA-assisted TTCM-aided MMSE-SDMA-OFDM system is given in Section II-B, followed by the introduction of the basic principles of the concatenated MMSE-GA MUD of Section III. Specifically, a review of the MMSE MUD is provided in Section III-A, the optimization metric of the GA MUD is derived in Section III-B, and Section III-C offers a detailed introduction to the concatenated MMSE-GA multiuser detection. In Section III-D, the novel BQM scheme is proposed, followed by the illustration of the IGA framework in Section III-E. Our simulation results are provided in Section IV, and the associated complexity issues are discussed in Section V. Finally, Section VI concludes our findings.

\section{SySTEM MODEL}

\section{A. SDMA MIMO Channel Model}

Fig. 1 shows an SDMA uplink MIMO channel model where each of the $L$ simultaneous mobile users employs a single transmit antenna, while the BSs receiver exploits $P$ antennas. At the $k$ th subcarrier of the $n$th OFDM symbol received by the $P$-element receiver antenna array, we have the received complex signal vector $\mathbf{x}[n, k]$, which is constituted by the superposition of the independently faded signals associated with the $L$ mobile users and contaminated by the additive white Gaussian noise

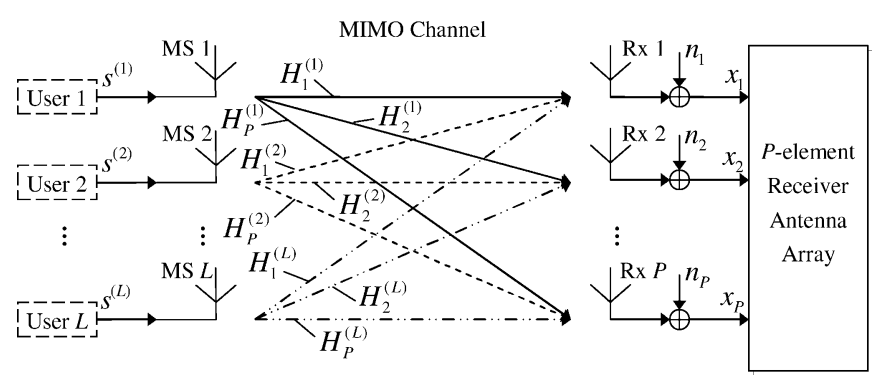

Fig. 1. Schematic of the SDMA uplink MIMO channel model [1], where each of the $L$ mobile users is equipped with a single transmit antenna and the BSs receiver is assisted by a $P$-element antenna front end.

(AWGN), expressed as

$$
\mathbf{x}=\mathbf{H} \mathbf{s}+\mathbf{n}
$$

where the $(P \times 1)$-dimensional vector $\mathbf{x}$, the $(L \times 1)$ dimensional vector $\mathbf{s}$, and the $(P \times 1)$-dimensional vector $\mathbf{n}$ are the received, transmitted, and noise signals, respectively. Here we have omitted the indices $[n, k]$ for each vector for the sake of notational convenience. Specifically, the vectors $\mathbf{x}, \mathbf{s}$, and $\mathbf{n}$ are given by

$$
\begin{aligned}
& \mathbf{x}=\left(x_{1}, x_{2}, \ldots, x_{P}\right)^{T} \\
& \mathbf{s}=\left(s^{(1)}, s^{(2)}, \ldots, s^{(L)}\right)^{T} \\
& \mathbf{n}=\left(n_{1}, n_{2}, \ldots, n_{P}\right)^{T} .
\end{aligned}
$$

The $(P \times L)$-dimensional matrix $\mathbf{H}$, which contains the frequency-domain channel transfer functions (FD-CHTF) of the $L$ users, is given by

$$
\mathbf{H}=\left(\mathbf{H}^{(1)}, \mathbf{H}^{(2)}, \ldots, \mathbf{H}^{(L)}\right)
$$

where $\mathbf{H}^{(l)}(l=1, \ldots, L)$ is the vector of the FD-CHTFs associated with the transmission paths from the $l$ th user's transmit antenna to each element of the $P$-element receiver antenna array, which is expressed as

$$
\mathbf{H}^{(l)}=\left(H_{1}^{(l)}, H_{2}^{(l)}, \ldots, H_{P}^{(l)}\right)^{T}, \quad l=\{1, \ldots, L\} .
$$

In (1)-(6), we assume that the complex signal $s^{(l)}$ transmitted by the $l$ th user has zero-mean and a variance of $\sigma_{l}^{2}$. The AWGN signal $n_{p}$ also exhibits a zero-mean and a variance of $\sigma_{n}^{2}$. The FD-CHTFs $H_{p}^{(l)}$ of the different receivers or users are independent, stationary, complex Gaussian distributed processes with zero-mean and unit variance [1].

\section{B. System Overview}

In Section II-A, we have briefly reviewed the SDMA MIMO channel model, as shown in Fig. 1. In Fig. 2, we present the schematic of the proposed concatenated MMSE-GA MUD aided SDMA-OFDM uplink system. At the transmitter end, as seen at the top of Fig. 2, the information bit sequences of the geographically separated $L$ simultaneous mobile users are forwarded to the TTCM [16] encoders, where they are encoded into symbols. The encoded signals $s^{(l)}(l=1, \ldots, L)$ are then 


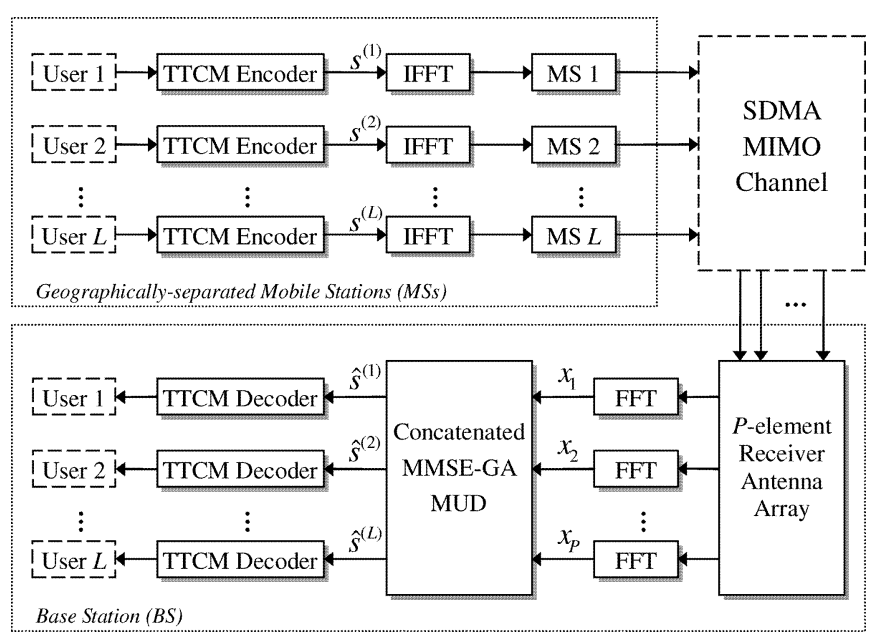

Fig. 2. Schematic of the MMSE-GA-concatenated multiuser detected SDMAOFDM uplink system.

forwarded to the OFDM-related inverse fast Fourier transform (IFFT) based modulator, which converts the frequency-domain signals to the time-domain modulated OFDM symbols. The OFDM symbols are then transmitted by the independent mobile stations (MSs) to the BS over the SDMA MIMO channel. Then, each element of the receiver antenna array shown at the bottom of Fig. 2 receives the superposition of the transmitted signals faded and contaminated by the channel and performs fast Fourier transform (FFT) based OFDM demodulation. The demodulated outputs $x_{p}(p=1, \ldots, P)$ seen in Fig. 2 are forwarded to the proposed concatenated MMSE-GA MUD for separating the different user's signals. The separated signals $\hat{s}^{(l)}(l=1, \ldots, L)$, namely the estimated versions of the transmitted signals, are then independently decoded by the TTCM decoders of Fig. 2 .

\section{Genetical Algorithm Assisted MMSE MULTIUSER DETECTION}

\section{A. MMSE MUD}

In the MMSE-based MUD, the estimates of the different user's transmitted signals are generated with the aid of the linear MMSE combiner. Specifically, the estimated signal vector $\hat{\mathbf{s}} \in \mathbb{C}^{L \times 1}$ generated from the transmitted signal s of the $L$ simultaneous users, as shown in Fig. 2, is obtained by linearly combining the signals received by the $P$ different receiver antenna elements with the aid of the array weight matrix as follows [1]:

$$
\hat{\mathbf{s}}_{\mathrm{MMSE}}=\mathbf{W}_{\mathrm{MMSE}}^{H} \mathbf{x}
$$

where the superscript $H$ denotes the Hermitian transpose, and $\mathbf{W}_{\text {MMSE }} \in \mathbb{C}^{P \times L}$ is the MMSE-based weight matrix given by [1]

$$
\mathbf{W}_{\text {MMSE }}=\left(\mathbf{H H}^{H}+\sigma_{n}^{2} \mathbf{I}\right)^{-1} \mathbf{H}
$$

where $\mathbf{I}$ is the identity matrix, and $\sigma_{n}^{2}$ is the AWGN noise variance.

\section{B. Optimization Metric for the GA MUD}

The optimum ML MUD [1] uses an exhaustive search for finding the most likely transmitted signals. For a ML-detection assisted SDMA-OFDM system supporting $L$ simultaneous users, a total of $2^{m L}$ metric evaluations has to be invoked, where $m$ denotes the number of bits per symbol (BPS) in order to detect the $L$-user symbol vector $\hat{\mathbf{s}}_{\mathrm{ML}}$ that consists of the most likely transmitted symbols of the $L$ users at a specific subcarrier, given by

$$
\hat{\mathbf{s}}_{\mathrm{ML}}=\arg \left\{\min _{\check{s} \in \mathcal{M}^{L}}\|\mathbf{x}-\mathbf{H} \check{\mathbf{s}}\|_{2}^{2}\right\}
$$

where the $(P \times 1)$-dimensional received signal vector $\mathbf{x}$ and the $(P \times L)$-dimensional frequency-domain channel transfer function (FD-CHTF) matrix $\mathbf{H}$ are defined by (2) and (5), respectively. The set $\mathcal{M}^{L}$ in (9), which is constituted by $2^{m L}$ trial-vectors, is formulated as

$$
\begin{aligned}
\mathcal{M}^{L} & \\
& =\left\{\check{\mathbf{s}}=\left(\check{s}^{(1)}, \check{s}^{(2)}, \ldots, \check{s}^{(L)}\right)^{T} \mid \check{s}^{(1)}, \check{s}^{(2)}, \ldots, \check{s}^{(L)} \in \mathcal{M}_{c}\right\}
\end{aligned}
$$

where $\mathcal{M}_{c}$ denotes the set containing the $2^{m}$ number of legitimate complex constellation points associated with the specific modulation scheme employed. The number of metric evaluations required for detecting the optimum vector increases exponentially with the number of users $L$.

Furthermore, the optimum ML-based decision metric of (9) may also be used in the GA-based MUD for the sake of detecting the estimated transmitted symbol vector $\hat{\mathbf{s}}_{\mathrm{GA}}$. In the context of the SDMA-OFDM system employing $P$ receiver antenna elements, the decision metric required for the $p$ th receiver antenna, namely the antenna-specific objective function [31], can be derived from (9), yielding

$$
\Omega_{p}(\mathbf{s})=\left|x_{p}-\mathbf{H}_{p} \mathbf{s}\right|^{2}
$$

where $x_{p}$ is the received symbol at the input of the $p$ th receiver at a specific OFDM subcarrier, and $\mathbf{H}_{p}$ is the $p$ th row of the channel transfer function matrix $\mathbf{H}$. Therefore, the decision rule for the optimum multiuser detector associated with the $p$ th antenna is to choose the specific $L$-symbol vector s, which minimizes the metric given in (11). Thus, the estimated transmitted symbol vector of the $L$ users based on the knowledge of the received signal at the $p$ th receiver antenna and a specific subcarrier is given by

$$
\hat{\mathbf{s}}_{\mathrm{GA}_{p}}=\arg \left\{\min _{\mathbf{s}}\left[\Omega_{p}(\mathbf{s})\right]\right\} .
$$

However, it follows from above derivation that we will have $P$ metrics in total for the $P$ receiver antennas. Since the channel impulse responses of each of the $P$ antennas are statistically independent, the $L$-symbol vector that is considered optimum at antenna 1 may not be considered optimum at antenna 2 , etc. In other words, this implies that a decision conflict is encountered, which may be expressed as

$$
\arg \left\{\min _{\mathbf{s}}\left[\Omega_{i}(\mathbf{s})\right]\right\}=\hat{\mathbf{s}}_{\mathrm{GA}_{i}} \neq \hat{\mathbf{s}}_{\mathrm{GA}_{j}}=\arg \left\{\min _{\mathbf{s}}\left[\Omega_{j}(\mathbf{s})\right]\right\}
$$


where $\forall i, j \in\{1, \ldots, P\}, i \neq j$. This decision conflict, therefore, leads to a so-called multiobjective optimization problem, since the optimization of the $P$ metrics may result in more than one possible $L$-symbol solution. A similar decision conflict resolution problem was studied in [34] in an attempt to reconcile the decision conflicts of multiple antennas resulting in a decision dilemma. In order to resolve this problem, we may adopt a similar approach and may amalgamate the $P$ number of antenna-specific $L$-symbol metrics into a joint metric as follows:

$$
\Omega(\mathbf{s})=\sum_{p=1}^{P} \Omega_{p}(\mathbf{s}) .
$$

Hence, the decision rule of the GA MUD is to find the specific estimated transmitted $L$-symbol vector $\hat{\mathbf{s}}_{\mathrm{GA}}$ that minimizes $\Omega(\mathbf{s})$ in (14) for every OFDM subcarrier considered.

\section{Concatenated MMSE-GA MUD}

The BER performance of the MMSE MUD is somewhat limited, since it is the total mean-square estimation error imposed by the different simultaneous users that is minimized, rather than directly optimizing the BER performance. Therefore, the MMSE-SDMA-OFDM system's BER performance may be potentially further improved with the aid of a concatenated GAaided MUD, which is capable of exploiting the output provided by the MMSE MUD of Section III-A in its initial population. For the sake of brevity, we will portray the philosophy of the proposed system in simplified terms. However, readers who are unfamiliar with GAs can consult [23] for a detailed description on GA-based optimization.

Fig. 3 shows a flowchart of the GA MUD employed in our multiuser SDMA-OFDM system.

1) Population Initialization: At the beginning of the GAbased search, an initial population consisting $X$ number of so-called individuals is created. In order to speed up the GA's search procedure, the first individual is created by making a hard decision at the output of the MMSE MUD of Section III-A. The other $X-1$ individuals are then generated by mutating ${ }^{1}$ the MMSE solution. Without loss of generality, an individual is represented by a symbol vector containing $L$ complex symbols, each of which belongs to one of the $L$ number of users at the specific subcarrier considered. Specifically, the $i$ th individual is expressed as

$$
\tilde{\mathbf{s}}_{i}^{(y)}=\left[\tilde{s}_{i, 1}^{(y)}, \tilde{s}_{i, 2}^{(y)}, \ldots, \tilde{s}_{i, L}^{(y)}\right], \quad i=\{1, \ldots, X\}
$$

where $\tilde{s}_{i, l}^{(y)}$ denotes the $l$ th $(l=1, \ldots, L)$ gene of the $i$ th individual. If for example a 4QAM modem constellation is used, each gene $\tilde{s}_{i, l}^{(y)}$ can be represented by one of the 4QAM constellation symbols, namely by $\tilde{s}_{i, l}^{(y)} \in \mathcal{M}_{c}$. The population consisting of $X$ number of individuals then forms the starting point of the optimization process, which is referred to as the $y=0$ th generation.

2) Fitness Value Evaluation: The GA's task is to find an individual, which is considered optimum or near-optimum

\footnotetext{
${ }^{1}$ The mutation process will be detailed later in this section.
}



Fig. 3. Structure of the GA MUD used in the multiuser SDMA-OFDM system.

in terms of minimizing the joint metric of (14) in the context of a $P$-antenna SDMA-OFDM system. For each individual, an associated objective score can be derived by evaluating (14). The objective score is then converted to a corresponding fitness value, which indicates the fitness of the specific individual. This fitness calculation is carried out in the "Fitness Value Evaluation" block of Fig. 3.

3) Mating Pool Creation: Based on the fitness values, $T$ number of individuals are selected for creating the socalled mating pool, following the principle of ParetoOptimality [23], [31]. This strategy favors the so-called non-dominated individuals and ignores the so-called dominated individuals [31]. Specifically, the $u$ th $L$-symbol individual is considered to be dominated by the $v$ th individual, if we have [35]

$$
\begin{array}{r}
\forall i \in\{1, \ldots, P\}: \Omega_{i}\left(\tilde{\mathbf{s}}_{v}^{(y)}\right) \leq \Omega_{i}\left(\tilde{\mathbf{s}}_{u}^{(y)}\right) \\
\wedge \exists j \in\{1, \ldots, P\}: \Omega_{j}\left(\tilde{\mathbf{s}}_{v}^{(y)}\right)<\Omega_{j}\left(\tilde{\mathbf{s}}_{u}^{(y)}\right)
\end{array}
$$


where $\Omega_{p}(\cdot)$ is defined by (11). If an individual is not dominated in the sense of (16) by any other individuals in the population, then it is considered to be nondominated. All the nondominated individuals are then selected and placed in the mating pool, which will have a size of $2<$ $T \leq X$ [31].

4) Selection: In order to evolve the population throughout the consecutive generations, the individuals in the mating pool are then selected as parents for producing offspring. The selection process is based on the socalled fitness-proportionate algorithm employed by the "fitness-proportionate selection" block of Fig. 3, which is widely used in the literature [36]. According to fitnessproportionate selection, each of the $T$ number of individuals in the mating pool is assigned a selection probability proportionate to its fitness value, which is calculated as follows. First, the so-called windowing-mapping [28] technique is invoked in order to get the fitness value $f_{i}^{(y)}$ associated with the $i$ th individual, which is given by

$$
f_{i}^{(y)}=\Omega_{T}^{(y)}-\Omega\left(\tilde{\mathbf{s}}_{i}^{(y)}\right)+c
$$

where

$$
\Omega_{T}^{(y)}=\max _{t \in\{1, \ldots, T\}}\left\{\Omega\left(\tilde{\mathbf{s}}_{t}^{(y)}\right)\right\}
$$

is the maximum objective score ${ }^{2}$ achieved by evaluating the $T$ number of individuals in the mating pool at the $y$ th generation, and $c$ is a small positive constant, which is used for the sake of ensuring the positiveness of $f_{i}^{(y)}$. Then, the fitness-proportionate probability of selection $p_{i}$ of the $i$ th individual can be formulated as

$$
p_{i}=\frac{f_{i}^{(y)}}{\sum_{j=1}^{T} f_{j}^{(y)}} .
$$

Therefore, the individuals having higher fitness values will be assigned higher selection probabilities, based on which $X / 2$ pairs of parents are selected. Moreover, during the selection process, the so-called incest prevention [37] technique can be invoked, which requires that the two individuals selected to form a pair of parents are different. This can effectively prevent the GA from premature convergence.

5) Crossover: For each pair of the $X / 2$ selected parents, a genetic operation referred to as crossover [24] is invoked. The crossover operation is a process during which some of the genes of a parent are exchanged with those of the other parent, thus creating two offspring. In our proposed GA MUD, the well-known uniform crossover [38] scheme corresponding to the "Uniform crossover" block of Fig. 3 is employed. For example, suppose each individual has $L=6$ genes. After two individuals are selected as parents from the mating pool, a binary crossover mask vector containing six randomly generated ones and zeros

\footnotetext{
${ }^{2}$ Note that the individual having the maximum objective score out of the pool of the $T$ candidates is considered as the worst solution in the context of the current mating pool, since the GA searches for the optimum solution which minimizes (14)
}

will be created. The genes are then exchanged between the selected pair of parents at positions associated with a one in the crossover mask, giving birth to two offspring.

6) Mutation: After the crossover operation is applied to each pair of parents, $X$ number of offspring are produced, which are then subject to the so-called mutation [24] operation, and some of the offspring's genes may be changed. Specifically, any gene of an offspring may be mutated to another legitimate gene under the control of the specific mutation strategy employed. Furthermore, the activation of the mutation process is governed by the so-called mutation probability $p_{m}$. Therefore, following the cross-over and mutation blocks illustrated in Fig. 3, the new population will be constituted by $X$ number of mutated offspring. It is worth pointing out that the mutation operation is critical to the success of the genetic evolution, since it ensures that sufficient diversity is maintained in the population, thus preventing the GA's search from being trapped at local optima. This will be further discussed in Section III-D.

7) Elitism: While the crossover and mutation operations offer the opportunity of improving the average fitness of the population, they do not guarantee that each of the offspring is better than their parents in terms of their fitness values. In other words, the better individuals associated with the higher fitness values found in the $y$ th generation may not be retained in the $(y+1)$ th generation. For the sake of ensuring that the high-fitness individuals are not lost from generation to generation, the best individual of the parents population is copied into the new population, replacing the worst offspring. This technique is known as elitism [24], as illustrated in Fig. 3.

The genetic operation cycle mentioned previously forms the basis of the GA-aided optimization, yielding an offspring population having an improved average fitness. This evolution continues until the generation index reaches its maximum. Then, the operation of the GA is terminated and the highest fitness individual of the last population will be considered as the final solution, which is the specific length- $L$ symbol vector that contains the detected transmitted symbols of the $L$ users at the specific OFDM subcarrier considered. If the population size $X$ and/or the number of generations $Y$ are sufficiently high, the GA's final solution approaches the optimum [31].

\section{Improved Mutation Scheme}

In the context of GA-based detection techniques, the efficiency of the mutation scheme employed is important for the success of the entire evolution procedure, since it provides a chance for the individuals of the current population to influence the forthcoming ones, so that new areas of the total search space may be explored and thus the chance of finding the optimum solution increases [39]. In the context of the GA-assisted multiuser SDMA-OFDM system, when the number of users $L$ increases or a high-throughput modulation scheme is used, the total search space consisting of $2^{m L}$ number of $L$-user symbol vectors would become excessive. In such cases, the role of mutation may become vital for the success of the overall 
system, since the GA may get trapped in local optima without appropriate assistance of the mutation scheme.

1) Conventional Uniform Mutation: According to the $M$-ary mutation employed by the GA MUD, each gene $\hat{s}^{(l)}(l=$ $1, \ldots, L)$ of a length- $L$ GA individual $\hat{\mathrm{s}}$ in the $X$-element population is represented by a specific symbol in $\mathcal{M}_{c}$. In other words, the $l$ th gene denotes the $l$ th user's estimated transmitted symbol at the subcarrier considered. During the genetic evolution, when a gene is subjected to mutation, it will be substituted by a different symbol in $\mathcal{M}_{c}$ based on a uniform mutation-induced transition probability $p_{m t}^{(i j)} 3$, which quantifies the probability of the $i$ th legitimate symbol becoming the $j$ th. For the sake of brevity, from now on we refer to this probability as the transition probability. Furthermore, we shall refer to the mutation scheme employing uniformly distributed $p_{m t}^{(i j)}$ values as uniform mutation (UM), which is a widely used conventional mutation scheme known in the literature [24], and was also employed by the GAs invoked in [31].

According to UM, the original gene will mutate to all the other candidate symbols in $\mathcal{M}_{c}$ with a same transition probability. However, this fixed uniform transition probability fails to reflect the realistic channel conditions that the system is subjected to. When considering a specific received symbol the adjacent constellation symbols are more likely to be the transmitted symbol, than the more distant ones. Hence, it may be more reasonable to consider only the neighboring symbols as the potential mutation candidates, and assign a modified biased transition probability, which is dependent on both the distance from the original symbol and on the signal-to-noise ratio (SNR). In other words, the GA's search space may be substantially reduced with the aid of a biased mutation, which pays less attention to the constellation points that are far from the received symbol. This is especially beneficial for the system employing high-throughput modulation schemes such as 16QAM, where the total search space is exponentially expanded as a function of the number of BPS compared to lower-throughput modems. In such a system, the UM-aided GA, which allows mutation to all legitimate symbols, may suffer from a slow convergence speed and might result in a high residual error floor, since a considerable portion of the GA's searching power may be wasted on mutating to highly unlikely gene candidates. By contrast, the biased mutation guided GA is expected to achieve a better performance, since it searches for the optimum solution in a more efficient way, as it will be demonstrated in Section III-D2.

2) Biased Q-Function Based Mutation (BQM): In this section, a novel mutation scheme will be presented which we call BQM. According to BQM, for an original gene to be mutated, an SNR-related biased transition probability $p_{m t}^{(i j)}$ will be assigned to each of the target candidate symbols in $\mathcal{M}_{c}$. The calculation of $p_{m t}^{(i j)}$ may be carried out with the aid of the widely known

\footnotetext{
${ }^{3}$ Note that the mutation probability $p_{m}$ is different from the probability $p_{m t}^{(i j)}$ of mutating to a specific symbol in $\mathcal{M}_{c}$. The former denotes the probability of how likely it is that a gene will mutate, while the latter specifies how likely it is that a specific symbol in $\mathcal{M}_{c}$ becomes the mutated gene.
}

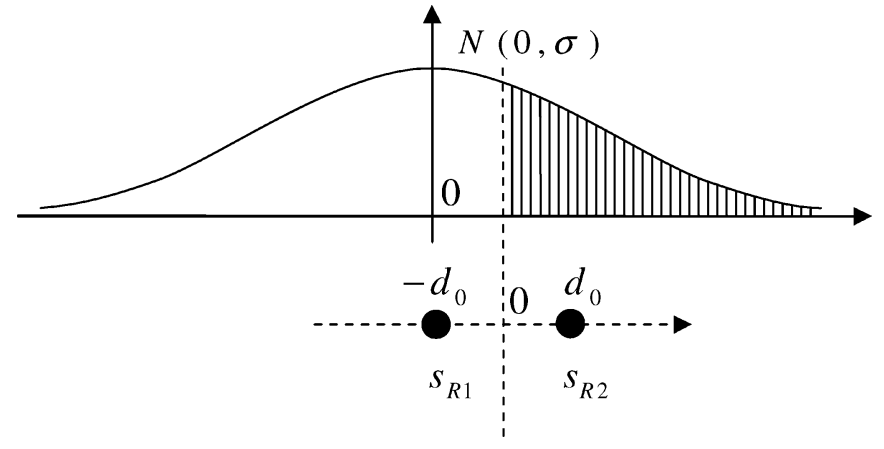

Fig. 4. Illustration of the 1-D transition probability $p_{m t}^{(i j)}$ for 4QAM.

$Q$-function [40]

$$
Q(x)=\frac{1}{\sqrt{2 \pi}} \int_{x}^{\infty} e^{-t^{2} / 2} d t, \quad x \geq 0 .
$$

For the sake of easy explanation, let us first consider a simple one-dimensional (1-D) scenario. In Fig. 4 we plotted the 1-D real component of the constellation symbols $\hat{s}_{i}^{(l)}$ in the context of the 4QAM modem constellation. The horizontal axis is then divided into two zones, each of which represents one specific 1-D constellation symbol $s_{R i}(i=1, \ldots, 2)$, as separated by the vertical dashed line of Fig. 4 . If $s_{R 1}$ is the original gene to be mutated, the Gaussian distribution $N(0, \sigma)$ may be centered at the position of $s_{R 1}$, where $\sigma$ is the noise variance at a given SNR level. In this specific example, $s_{R 2}$ is the only mutation target, and the 1-D transition probability of mutating from $s_{R 1}$ to $s_{R 2}$; i.e., $p_{m t}^{\left(\frac{12}{2}\right)}$, is characterized by the shadow area shown in Fig. 4, which is given by

$$
p_{m t}^{(12)}=Q\left(\frac{d_{0}}{\sigma}\right)
$$

where $d_{0}$ is half of the distance between the neighboring constellation symbols. Similarly, we have

$$
p_{m t}^{(21)}=Q\left(\frac{d_{0}}{\sigma}\right) \text {. }
$$

We also have a certain probability for the original gene to remain unchanged, which can also be expressed in terms of the Gaussian distribution as

$$
p_{m t}^{(11)}=p_{m t}^{(22)}=1-Q\left(\frac{d_{0}}{\sigma}\right) .
$$

The corresponding two-dimensional (2-D) symbol transition probability $p_{m t}^{(i j)}$ can be derived by combining the 1-D real and imaginary transition probabilities. ${ }^{4}$ For the specific $1-D-b a s e d$ 4QAM example of Fig. 4, we plot the corresponding 2-D constellation in Fig. 5. In Fig. 5, for instance, the 2-D transition probability of mutating from the constellation symbol $\hat{s}_{1}^{(l)}$ to $\hat{s}_{2}^{(l)}$, namely $p_{m t}^{(12)}$, can be calculated by multiplying the two

\footnotetext{
${ }^{4}$ Note that the 1-D transition probability $p_{m t}^{(i j)}$ is different from the transition probability $p_{m t}^{(i j)}$, which is based on the 2-D constellation symbols.
} 


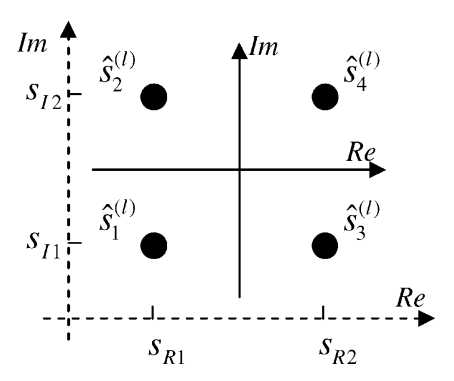

Fig. 5. Illustration of the 2-D transition probability $p_{m t}^{(i j)}$ for 4QAM, which is the product of the relevant 1-D transition probabilities. $s_{R i}$ and $s_{I i}(i=1,2)$ denote the 1-D constellation symbols in the context of the real and imaginary components of the 4QAM constellation symbols, respectively.

relevant 1-D transition probabilities according to ${ }^{5}$

$$
p_{m t}^{(12)}=p_{m t}^{(11)} \cdot p_{m t}^{(12)}=\left(1-Q\left(\frac{d_{0}}{\sigma}\right)\right) \cdot Q\left(\frac{d_{0}}{\sigma}\right)
$$

while the associated 2-D probability of remaining in the current state is

$$
p_{m t}^{(11)}=p_{m t}^{(11)} \cdot p_{m t}^{(11)}=\left(1-Q\left(\frac{d_{0}}{\sigma}\right)\right)^{2}
$$

However, when mutation takes place during the evolution, the mutating gene or constellation symbol should not be allowed to be mutated to itself. Hence, the effect of the probability of mutating a symbol to itself should be removed. This can be achieved by normalizing the 2-D transition probability $p_{m t}^{(i j)}$ $(i \neq j)$ with the aid of the original gene's probability of remaining unchanged, namely $p_{m t}^{(i i)}$, following the principles of conditional probability theory [41].

For higher throughput modems, for example for 16QAM and 64QAM, the same algorithm can be invoked for calculating the corresponding 1-D and 2-D transition probabilities. Moreover, the proposed BQM scheme can be readily extended to $M$-dimensional (MD) constellations, since the MD transition probability associated with a specific MD symbol can be readily derived upon multiplying the $M$ number of corresponding 1-D transition probabilities.

3) Simplified BQM: Furthermore, the proposed BQM scheme can be effectively simplified, when only a subset of all the theoretically possible mutation target symbols are considered. More precisely, for the original gene subjected to mutation, we may only consider its adjacent neighboring constellation symbols as mutation target candidates, since the original transmitted symbol is less unlikely to be corrupted to a relatively distant constellation symbol.

An example of the simplified BQM designed for 16QAM is provided in Fig. 6. As shown in Fig. 6, for example, we assume that $\hat{s}_{1}^{(l)}$ is the original gene subjected to mutation,

\footnotetext{
${ }^{5}$ Note that the superscripts $i$ and $j$ of the 2-D transition probability $p_{m t}^{(i j)}$ denote the 2-D constellation symbols $\hat{s}_{i}^{(l)}$ and $\hat{s}_{j}^{(l)}$, while the underlined superscripts $i$ and $j$ of the 1-D transition probability $p_{m t}^{(i j)}$ represent the 1-D constellation symbols $s_{R i}$ and $s_{R j}$, respectively.
}



Fig. 6. Example of the simplified BQM for 16QAM.

TABLE I

Possible Transition Probability VALUES FOR THE CNUM SCHEME

\begin{tabular}{|c|c|}
\hline Modem & Transition probability value set \\
\hline 4QAM & $1 / 2$ \\
\hline 16QAM & $1 / 2,1 / 3,1 / 4$ \\
\hline 64QAM & $1 / 2,1 / 3,1 / 4$ \\
\hline
\end{tabular}

and $\hat{s}_{i}^{(l)}(i=2, \ldots, 9)$ represents the adjacent neighbors of $\hat{s}_{1}^{(l)}$, while the symbols represented by the dashed-line circles are ignored. Therefore, the GA's entire search space is reduced. Moreover, the search space can be further reduced when we only consider the nearest neighbors of $\hat{s}_{1}^{(l)}$ as the legitimate mutation candidates, namely the symbols $\hat{s}_{i}^{(l)}(i=3,5,6,8)$, which are shown in grey in Fig. 6. Each of these symbols is then assigned an equal 2-D transition probability $p_{m t}^{(1 j)}=1 / 4(j=3,5,6,8)$, while all other constellation symbols shown in white are neglected. Since the transition probability for each selected mutation candidate is set to be identical, the BQM scheme is simplified to a new scheme similar to UM which we refer to as the closest-neighbor uniform mutation (CNUM) scheme. Note that in CNUM, the corresponding transition probability value is only dependent on the location of the original gene. For instance, if the original gene is located in one of the four corners of the constellation map plotted in Fig. 6, the relevant transition probability $p_{m t}^{(i j)}$ becomes $1 / 2$, since in this case only two nearest-neighbor symbols exist. The CNUM-related transition probability values of the different modems are summarized in Table I. Hence, by introducing the simplified BQM scheme or the CNUM arrangement, the computational complexity of BQM can be reduced.

\section{E. Iterative GA MUD}

The performance of the multiuser SDMA-OFDM system employing the BQM-assisted GA MUD can be further improved when an enhanced iterative detection architecture is utilized. In the literature, iterative techniques such as SIC [1]-[6], and PIC [1], [2], [6], [7], have been designed for multiuser OFDM systems. Following the philosophy of iterative detections, we 


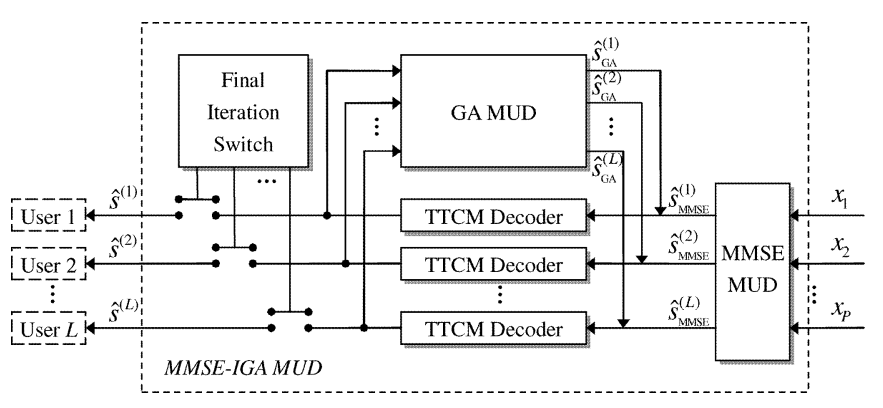

Fig. 7. Structure of the MMSE-initialized iterative GA MUD used at the BS.

propose an MMSE-initialized iterative GA (IGA) MUD for multiuser SDMA-OFDM systems.

The detailed structure of the IGA MUD is outlined in Fig. 7. Specifically, the received length- $P$ symbol vector $\mathrm{x}$ of (2) is first detected by the MMSE MUD, which outputs the $L$ MMSE-detected symbols $\hat{s}_{\mathrm{MMSE}}^{(l)}(l=1, \ldots, L)$ of the $L$ users and forwards them to $L$ number of independent TTCM decoders. The TTCM-decoded $L$-symbol vector, which is more reliable than the MMSE MUD's output, is then fed into the concatenated GA MUD for assisting the creation of the initial population. Then the genetically enhanced output symbol vector $\hat{\mathbf{s}}_{\mathrm{GA}}$, which may be expected to become more reliable, will be fed back to the TTCM decoders in order to further improve the signal's quality, invoking a number of iterations. Following the last iteration, the final GA solution will be decoded by the TTCM decoders, and the hard-decision version of the estimated information bits of the $L$ independent users is forwarded to the output, which is only enabled at the final iteration by the switch seen in Fig. 7.

Therefore, two improvements have been achieved by the MMSE-IGA MUD. First, a more accurate initial knowledge of the transmitted signals, namely the output of the TTCM decoders rather than that of the MMSE MUD, is supplied for the GA MUD. This reliable improvement, therefore, offers a better starting point for the GA's search. Second, the iterative processing ensures that the detected $L$-user symbol vector can be optimized in two dimensions. During every iteration, on one hand, each $L$-symbol vector at a specified subcarrier slot is optimized by the GA in the context of the user domain. On the other hand, the entire TTCM-coded frame of each user is optimized by the TTCM decoder in the context of the TTCM-related codeword domain, or more specifically the frequency domain. Therefore, as the iterative processing continues, an information exchange takes place between the two domains, and thus an improved system performance may be expected.

\section{Simulation RESUltS}

In this section, we characterize the performance of the proposed TTCM-assisted concatenated MMSE-GA multiuser detected SDMA-OFDM system. The channel was assumed to be "OFDM symbol-invariant," implying that the taps of the impulse response were assumed to be constant for the duration of one OFDM symbol, but they were faded at the beginning of each OFDM symbol [1]. The simulation results were obtained over
TABLE II

VARIOUS TECHNIQUES AND PARAMETERS USED IN OUR SIMULATIONS

\begin{tabular}{|c|l|l|}
\hline \multirow{5}{*}{} & Population initialization method & MMSE \\
\cline { 2 - 3 } & Mating pool creation strategy & Pareto-Optimality \\
\cline { 2 - 3 } & Selection method & Fitness-Proportionate \\
\cline { 2 - 3 } & Cross-over & Uniform cross-over \\
\cline { 2 - 3 } & Mutation & UM or BQM \\
\cline { 2 - 3 } & Mutation probability & 0.1 \\
\cline { 2 - 3 } & Elitism & Enabled \\
\cline { 2 - 3 } & Incest prevention & Enabled \\
\cline { 2 - 3 } & Population size & Varied \\
\cline { 2 - 3 } & Generations & Varied \\
\cline { 2 - 3 } & IGA iterations & Varied \\
\hline \multirow{5}{*}{ OFDM } & Subcarriers & 512 \\
\cline { 2 - 3 } & Cyclic prefix & 64 \\
\hline \multirow{5}{*}{ Channel } & CIRs & SWATM [1] \\
\cline { 2 - 3 } & Paths & $3.235 \times 10^{-5}$ \\
\cline { 2 - 3 } & Maximum path delay & \\
\cline { 2 - 3 } & Normalized Doppler frequency & SW \\
\hline
\end{tabular}

a channel characterized by the three-path short wireless asynchronous transfer mode (SWATM) CIR given in [1, p. 78]. The maximum path delay is $48.9 \mathrm{~ns}$, while each of the paths experiences independent Rayleigh fading having the same normalized Doppler frequency of $1.235 \times 10^{-5}$. The OFDM modem employed $K=512$ subcarriers and a cyclic prefix of 64 samples, which is longer than the maximum channel delay spread. If not explicitly stated, it was assumed that the channels' transfer functions were perfectly known. For the iterative TTCM scheme [16] used, the code memory $\nu$ was fixed to three, the generator polynomial expressed in octal format was $(136)$, and the codeword length and channel interleaver depth were fixed to 1024. For each of the various schemes evaluated, a similar number of overall TTCM decoding iterations was maintained so that the total TTCM-related complexity remained approximately the same for all systems. Table II summarizes the various techniques and parameters used in our simulations.

\section{A. BQM Versus $U M$}

Fig. 8 shows the BER performance achieved by the various schemes considered. The performance of the TTCM-assisted MMSE-SDMA-OFDM system, the TTCM-assisted optimum ML-detected system, and the uncoded single-user scheme employing a single receiver when communicating over an AWGN channel are also provided for reference, respectively. The numbers in the round brackets seen in the legends of Fig. 8 denote the associated number of IGA MUD iterations. From the results of Fig. 8, two conclusions may be made. First, improved performance can be achieved when the GA commences its operation from a better initial population, regardless of the different mutation schemes used. For example, at the same GA complexity, the single-iteration IGA MUD assisted systems outperformed 




Fig. 8. BER versus $E_{b} / N_{0}$ performance comparison of the TTCM-assisted MMSE-GA-SDMA-OFDM or MMSE-IGA-SDMA-OFDM system using UM or BQM, while employing a 4QAM scheme for transmission over the SWATM channel, where $L=6$ users are supported with the aid of $\mathbf{P}=\mathbf{6}$ receiver antenna elements, respectively. The GA-related system configuration are given in Table II.

their noniterative GA aided counterparts, since the initial GA population of the former systems were created based on the first-iteration outputs of the TTCM decoders, rather than on the less reliable MMSE MUD regardless, whether UM or BQM was employed. Secondly, the BQM-aided systems achieved a better performance than the UM-aided schemes, since BQM is more efficient in guiding the GA towards the optimum solution, as seen in Fig. 8. Furthermore, the system employing the BQM-aided two-iteration IGA MUD was capable of achieving a virtually indistinguishable performance from that of the optimum ML-aided system.

When a high-throughput modem such as, for example, 16QAM, is employed, BQM may significantly outperform UM, as evidenced in Fig. 9, where six users were supported. ${ }^{6}$ As seen in Fig. 9, even when the IGA was employed, the UM-aided scheme yielded a high residual error floor due to the less efficient mutation strategy. By contrast, BQM significantly improved the GA's performance by lowering the error floor by about two orders of magnitude. Furthermore, when the number of IGA MUD iterations was increased, the performance of the BQMaided system can be dramatically improved, while the UM-aided scheme still suffered from an error floor. This suggests that the BQM-aided scheme is capable of substantially benefitting from both a more meritorious initial GA population, and from a higher number of IGA MUD iterations. On one hand, the improved initial population provides a good starting point for the GA, thus assisting the BQM, which in turn benefits the entire detection process, resulting in a substantial performance improvement. On the other hand, the iterative processing invoked by the IGA MUD further enhances the system's performance with the aid

\footnotetext{
${ }^{6}$ Note that in this case the associated complexity of the ML-aided scheme is as high as on the order of $O\left(2^{m L}\right)=O\left(2^{4 \cdot 6}\right)=O(16,777,216)$, which imposes an excessive complexity, and hence cannot be simulated.
}

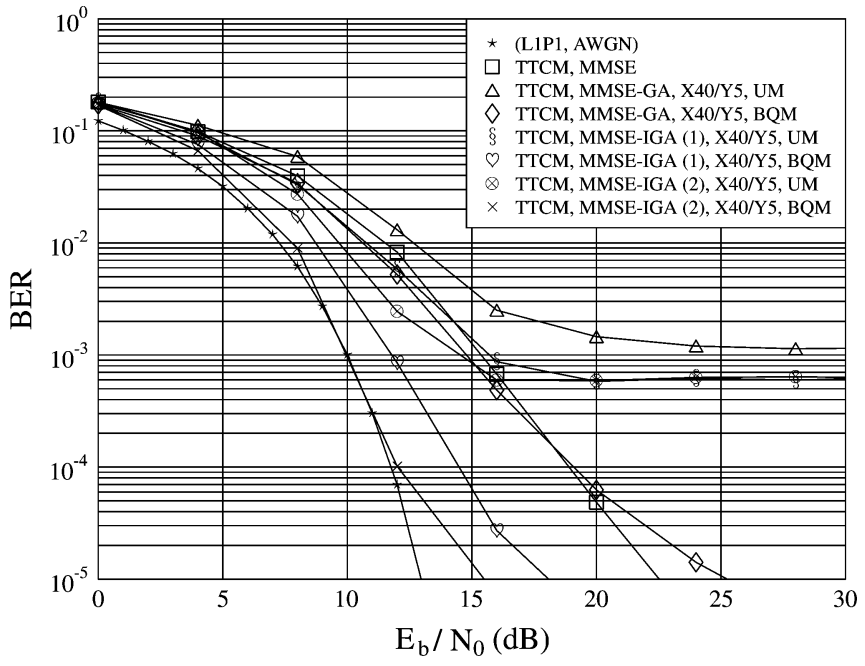

Fig. 9. BER versus $E_{b} / N_{0}$ performance comparison of the TTCM-assisted MMSE-GA-SDMA-OFDM or MMSE-IGA-SDMA-OFDM system using UM or BQM, while employing a 16QAM scheme for transmission over the SWATM channel, where $L=6$ users are supported with the aid of $L=6$ receiver antenna elements, respectively. The GA-related system configuration are given in Table II.

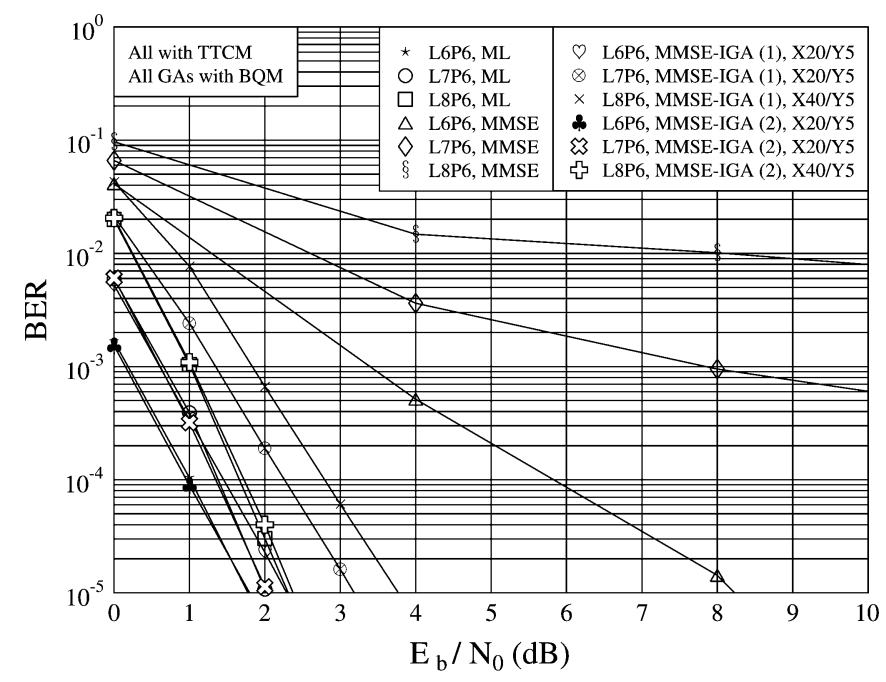

Fig. 10. BER versus $E_{b} / N_{0}$ performance comparison of the TTCM-assisted MMSE-IGA-SDMA-OFDM system using BQM, while employing a 4QAM scheme for transmission over the SWATM channel, where $L=6,7,8$ users are supported with the aid of $P=6$ receiver antenna elements, respectively. The GA-related system configuration are given in Table II.

of the 2-D optimization, as discussed in Section III-E, since the beneficial information exchange between the user domain and the frequency domain assists both the GA MUD and the TTCM decoder in eliminating more and more errors found in the received signal, as the iterative procedure continues.

\section{B. Overloaded BQM-IGA}

Fig. 10 shows the performance achieved by the BQM-IGA aided TTCM-SDMA-OFDM system using 4QAM, when six, seven, and eight users are supported by six receiver antenna elements, respectively. It is seen in Fig. 10 that in the so-called overloaded scenarios, where the number of users exceeds the 


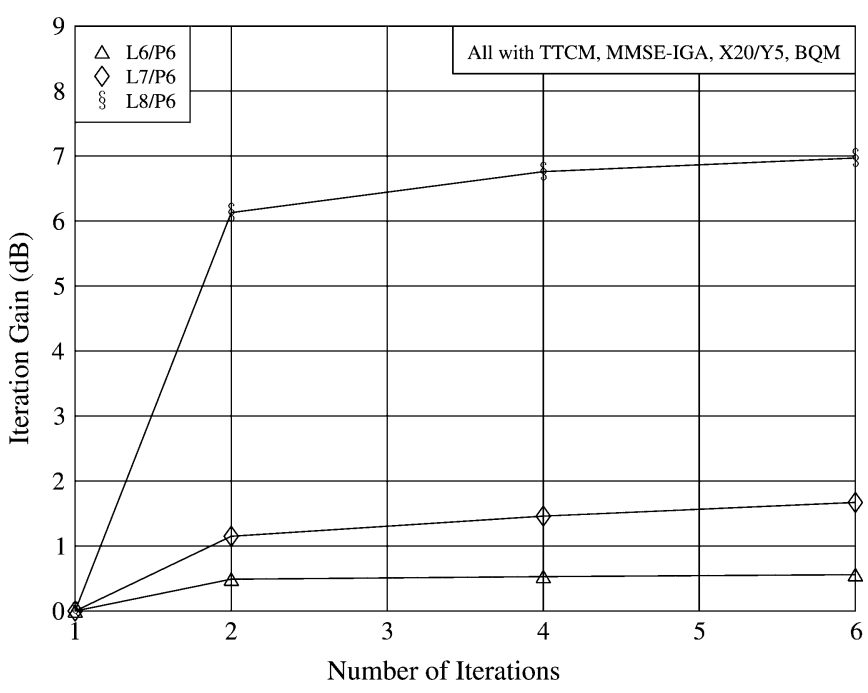

Fig. 11. Iteration gain at the BER of $10^{-5}$ versus number of IGA MUD iterations performance of the TTCM-assisted MMSE-IGA-SDMA-OFDM system using BQM, while employing a 4QAM scheme for transmission over the SWATM channel, where $L=6,7,8$ users are supported with the aid of $P=6$ receiver antenna elements, respectively. The GA-related system configuration are given in Table II.

number of receiver antenna elements, the linear MMSE MUD suffered from an insufficient degree of freedom for separating the different users, since the high number of users incurred excess multiuser interference (MUI). This results in a significant performance degradation in the context of the system using the MMSE MUD, when the number of users increased from six to eight, as observed in Fig. 10. However, in such cases, the system employing the proposed BQM-IGA MUD was still capable of maintaining a near-ML performance. For example, when we had $L=8$, the two-iteration based BQM-IGA MUD reduced the BER measured at $3 \mathrm{~dB}$ by four orders of magnitude in comparison to the MMSE-aided benchmarker system, as shown in Fig. 10. This result characterizes the robustness of the BQMIGA MUD, which has successfully suppressed the high MUI experienced in overloaded scenarios.

Fig. 11 shows the iteration gain achieved by the BQMIGA assisted TTCM-MMSE-SDMA-OFDM system employing 4QAM at the BER of $10^{-5}$, while using different number of IGA MUD iterations. The iteration gain is defined here as the $E_{b} / N_{0}$ difference of the systems employing different number of IGA MUD iterations measured at the BER of $10^{-5}$ in comparison to the baseline system employing a single IGA MUD iteration. It is found in Fig. 11 that when more users are supported, higher iteration gains may be obtained by iterative detection. For example, a gain of about $6 \mathrm{~dB}$ was attained by the eight-user system at the second IGA MUD iteration, while that attained by the sixuser system was only about $0.5 \mathrm{~dB}$. Furthermore, as the number of iterations was increased from two to six, the former scheme provided a further gain of about $1 \mathrm{~dB}$, while no explicit gain was achieved by the latter arrangement, as shown in Fig. 11. It is also seen in Fig. 11 that most of the achievable iteration gain has been attained at the second IGA MUD iteration for all the schemes.

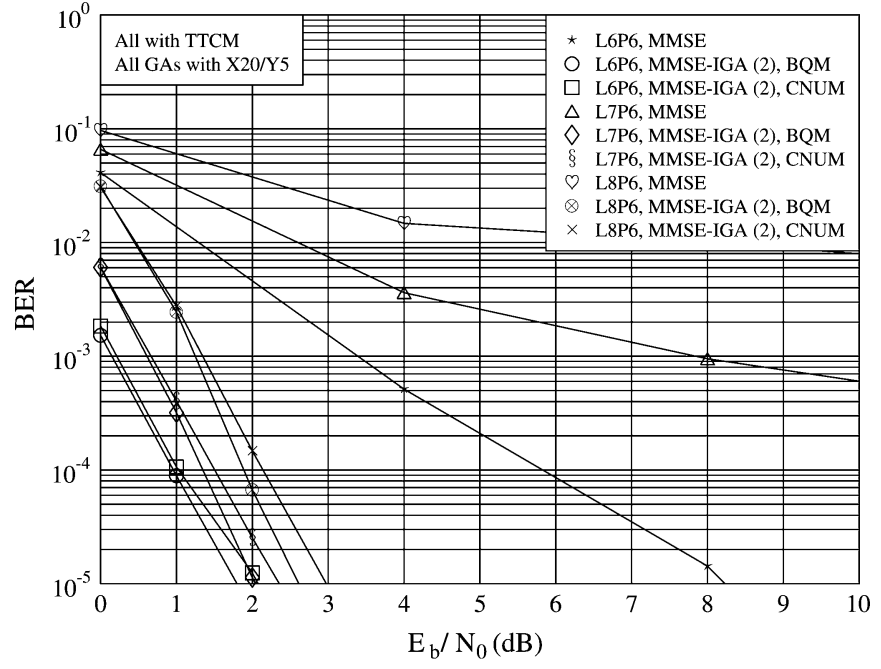

Fig. 12. BER versus $E_{b} / N_{0}$ performance comparison of the TTCM-assisted MMSE-IGA-SDMA-OFDM system using BQM or CNUM, while employing a 4QAM scheme for transmission over the SWATM channel, where $L=6,7,8$ users are supported with the aid of $P=6$ receiver antenna elements, respectively. The GA-related system configuration are given in Table II.

\section{BQM Versus CNUM}

As discussed in Section III-D3, the BQM scheme may be simplified to the CNUM arrangement, which mutates to only one of the closest neighbors of the original gene, thus reducing the complexity of BQM. However, CNUM does not necessarily degrade the system's performance dramatically. Fig. 12 provides a performance comparison of BQM and CNUM in the fully loaded and overloaded scenarios. As observed in Fig. 12, the BQM-IGA aided system achieved slightly better performance than its CNUM-IGA aided counterpart. This may suggest that in such scenarios the CNUM scheme may become an attractive alternative to the $\mathrm{BQM}$ scheme for the sake of further decreasing the complexity imposed.

\section{Performance Under Imperfect Channel Estimation}

As a further investigation, we provide the simulation results generated in the scenario where the channel state information (CSI) was assumed to be imperfect. The estimated CIRs $\hat{h}_{i}$ were generated by adding random Gaussian noise to the true CIR taps $h_{i}$ as

$$
\hat{h}_{i}[n]=h_{i}[n]+\sqrt{\frac{\sigma_{n}^{2}}{\varepsilon}} n_{i}[n], \quad i=1, \ldots, \mathcal{L}
$$

where $\varepsilon$ is the effective noise factor, $\sigma_{n}^{2}$ is the noise variance at the specific SNR level, $n_{i}$ is an AWGN sample having zeromean and a variance of unity, $\mathcal{L}$ is the number of CIR taps, and $[n]$ denotes the $n$th OFDM symbol. In the scenarios associated with imperfect CIRs, $\varepsilon$ was set to 64 , and $\mathcal{L}$ was set to three for the three-path SWATM channel used. In this case, the effective noise power added to the true CIR taps during each OFDM symbol for the sake of simulating imperfect channel estimation was $\sigma_{n}^{2} \cdot \mathcal{L} / \varepsilon=\sigma_{n}^{2} \times 4.69 \%$.

Our performance comparison of the proposed BQMIGA aided TTCM-MMSE-SDMA-OFDM system under the 


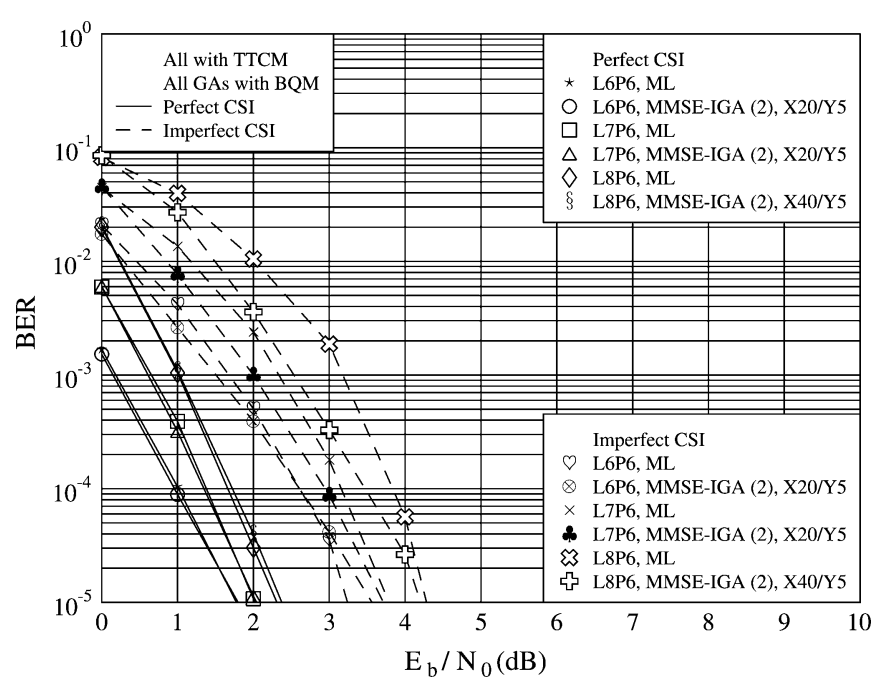

Fig. 13. BER versus $E_{b} / N_{0}$ performance comparison of the TTCM-assisted MMSE-IGA-SDMA-OFDM system using BQM, while employing a 4QAM scheme for transmission over the SWATM channel, where $L=6,7,8$ users are supported with the aid of $P=6$ receiver antenna elements, respectively. The GA-related system configuration is given in Table II.

assumptions both of perfect and imperfect CSI is provided in Fig. 13. As seen in Fig. 13, the proposed system was capable of attaining an acceptable performance even without accurate channel knowledge. Moreover, it was found that when imperfect channel estimation was assumed, the BQM-IGA aided system outperformed its ML-aided counterpart, especially in the scenarios associated with higher user loads. This phenomenon may be explained as follows. When the CSI is imperfect, the MLdetected signal becomes less reliable than that detected in the scenario benefitting from perfect CSI. The relatively unreliable output of the ML MUD may readily mislead the TTCM decoder due to error propagation, resulting in a performance degradation. However, the detrimental effects of imperfect CSI may be mitigated by the proposed IGA MUD. More specifically, the IGA MUD optimizes the detected signal in two dimensions, namely in both the user domain as well as in the frequency domain, as discussed in Section III-E. The beneficial information exchange offered by the IGA MUD between the two domains may effectively assist the concatenated detection-decoding procedure in counteracting the detrimental effects of imperfect channel estimation. This results in better system performance in comparison to that achieved by the ML-aided system. Furthermore, when a higher number of users had to be supported, the ML-aided system using imperfect CSI suffered more from inaccurate multiuser detection, while a more robust behavior was exhibited by the IGA-aided system, as shown in Fig. 13.

\section{COMPLEXITY ANALYSIS}

Compared to the conventional UM scheme, BQM is capable of significantly improving the GA's performance, especially in high-throughput or high-SNR scenarios, as discussed in Section IV-A. Furthermore, this performance improvement was achieved at the cost of a modest complexity increase and a modest memory requirement. At different SNR levels, for each of the $2^{m}$ constellation symbols, a specific set containing $\left(2^{m}-1\right)$ number of normalized 2-D transition probabilities has to be created. However, this only imposes a modest "once-forall" calculation, since we can derive the associated transition probabilities with the aid of offline experiments for a number of typical SNR levels, where the calculated data can be stored in the base station's memory, hence incurring no further computational complexity. Furthermore, by introducing the simplified BQM scheme of Section III-D3, the associated complexity and memory cost may be dramatically reduced, especially for high-throughput modems such as 16QAM or 64QAM, since the number of mutation target candidates decreases, and thus fewer transition probability calculations are required. Moreover, if the CNUM scheme is employed, the associated complexity can be further decreased since, in this case, there is no need to calculate the transition probabilities, which are already available in Table I. This may significantly reduce the associated complexity and memory requirement, while still maintaining performance similar to that of the BQM scheme as seen in Fig. 12.

As suggested in Sections IV-A and IV-B, the performance of the IGA-aided system can be further improved when the number of IGA MUD iterations or the GA's parameters, such as the population size, are increased. These will result in a further increased complexity which, however, may still be significantly lower than that imposed by the ML-aided scheme. In Table III we compare the computational complexity imposed by the different MUDs in terms of the number of complex additions and multiplications on a per user basis, where the number of receiver antenna elements was fixed to $P=6$, and an $E_{b} / N_{0}$ value of $3 \mathrm{~dB}$ was assumed. At a specific user load, we always selected the IGA-aided scheme for comparison, which achieved an almost indistinguishable performance compared to the corresponding ML-aided system. As shown in Table III, the complexity of the ML MUD is significantly higher than that of the MMSE MUD or the IGA MUD, especially in highly overloaded scenarios. On the other hand, the IGA MUD reduced the BER by up to five orders of magnitude in comparison to the MMSE MUD at a moderate complexity. It is also worth pointing out that the IGA MUD's complexity remained nearly identical at different $E_{b} / N_{0}$ values according to our simulation results, which are not included here due to space limitations.

\section{SUMMARY AND DISCUSSION}

From the investigations conducted, we conclude that the GA-assisted TTCM-aided MMSE-SDMA-OFDM system is capable of achieving performance similar to that of the optimum ML-assisted TTCM-SDMA-OFDM system. The novel BQM scheme is capable of improving the GA's search at a modest complexity increase, thereby significantly increasing the chances of finding the optimum GA solution in highSNR and/or high-throughput scenarios. On the other hand, the 2-D optimization provided by the proposed IGA MUD has been shown to be beneficial for the SDMA-OFDM system in both the frequency and user domains. Finally, the scheme that combines BQM with the IGA MUD yields the best performance 
TABLE III

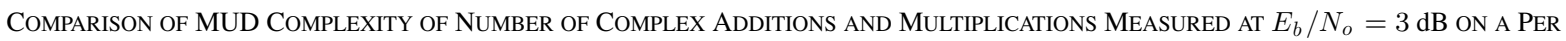
USER BASIS IN THE 4QAM TTCM-SDMA-OFDM SYSTEM

\begin{tabular}{|c|c|c|c|c|c|c|c|c|c|}
\hline \multirow{2}{*}{ MUD } & \multicolumn{4}{|c|}{$L=6$} & \multicolumn{3}{c|}{$L=7$} & \multicolumn{3}{c|}{$L=8$} \\
\cline { 2 - 10 } & + & $\times$ & BER & + & $\times$ & BER & + & $\times$ & BER \\
\hline ML & $2.8 \times 10^{4}$ & $2.7 \times 10^{4}$ & $1.8 \times 10^{-7}$ & $1.1 \times 10^{5}$ & $1.1 \times 10^{5}$ & $5.1 \times 10^{-7}$ & $4.3 \times 10^{5}$ & $4.2 \times 10^{5}$ & $8.5 \times 10^{-7}$ \\
\hline IGA & $8.1 \times 10^{2}$ & $7.9 \times 10^{2}$ & $2.2 \times 10^{-7}$ & $8.7 \times 10^{2}$ & $8.5 \times 10^{2}$ & $6.2 \times 10^{-7}$ & $1.8 \times 10^{3}$ & $1.7 \times 10^{3}$ & $9.8 \times 10^{-7}$ \\
\hline MMSE & $7.1 \times 10^{1}$ & $9.0 \times 10^{1}$ & $1.5 \times 10^{-3}$ & $7.1 \times 10^{1}$ & $8.8 \times 10^{1}$ & $7.5 \times 10^{-3}$ & $7.1 \times 10^{1}$ & $8.7 \times 10^{1}$ & $2.2 \times 10^{-2}$ \\
\hline
\end{tabular}

in all scenarios considered, while maintaining a significantly lower computational complexity than that imposed by the MLassisted system, especially when the number of users is high, as evidenced by Table III. Furthermore, the proposed BQM-IGA MUD is capable of achieving a near-optimum performance in the so-called over-loaded scenarios, where the number of users supported is higher than the number of receiver antenna elements. In this case the classic linear MMSE MUD results in an excessive residual BER, since the grade of freedom becomes insufficiently high. Moreover, when the channel estimation is imperfect, the proposed scheme still provides a satisfactory performance.

The system parameters of the IGA framework, such as the number of TTCM iterations the number of IGA MUD iterations, and the GA-related parameter settings, are all readily configurable, enabling us to strike an attractive tradeoff between the achievable performance and the complexity imposed. For specific scenarios, the TTCM scheme used in the system can also be conveniently substituted by other FEC schemes-for example, the TC codes. Therefore, the facility provided by the proposed IGA MUD may make it relavent to applications in multimode terminals, where good performance, low complexity, and easy flexibility are all important criteria.

It is also worth pointing out that the proposed BQM-IGA MUD can be readily incorporated into multiuser CDMA systems such as those of [31]. In this case, the initial detected signal supplied to the GA MUD for creating the first population is provided by the bank of matched filters installed at the CDMA BS, rather than by the MMSE MUD. However, the BQM scheme may remain unchanged. Our future research will consider various overloaded sphere decoding aided SDMA-OFDM systems.

\section{REFERENCES}

[1] L. Hanzo, M. Münster, B. J. Choi, and T. Keller, OFDM and MC-CDMA for Broadband Multi-User Communications, WLANs and Broadcasting. Piscataway, NJ: IEEE Press, New York: Wiley, 2003.

[2] S. Verdu, Multiuser Detection. Cambridge, U.K.: Cambridge Univ. Press, 1998.

[3] C. Z. W. H. Sweatman, J. S. Thompson, B. Mulgrew, and P. M. Grant, "A comparison of detection algorithms including BLAST for wireless communication using multiple antennas," in Proc. IEEE Int. Symp. Personal, Indoor and Mobile Radio Communications, vol. 1, London, U.K., Sep. 2000, pp. 698-703.

[4] P. Vandenameele, L. van Der Perre, M. Engels, B. Gyselinckx, and H. D. Man, "A novel class of uplink OFDM/SDMA algorithms: A statistical performance analysis," in Proc. IEEE Vehicular Technology Conf., vol. 1, Amsterdam, The Netherlands, Sep. 1999, pp. 324-328.

[5] P. Vandenameele, Space Division Multiple Access for Wireless Local Area Networks. London, U.K.: Kluwer, 2001.
[6] M. Münster and L. Hanzo, "Co-channel interference cancellation techniques for antenna array assisted multiuser OFDM systems," in Proc. IEEE 3G'2000 Conf., vol. 1, London, U.K., Mar. 2000, pp. 256260.

[7] M. Sellathurai and S. Haykin, "A simplified diagonal BLAST architecture with iterative parallel-interference cancellation receivers," in Proc. IEEE Int. Conf. Communications, vol. 10, Helsinki, Finland, Jun. 2001, pp. 3067-3071.

[8] P. Vandenameele, L. van Der Perre, M. Engels, B. Gyselinckx, and H. D. Man, "A combined OFDM/SDMA approach," IEEE J. Sel. Areas Commun., vol. 18, no. 11, pp. 2312-2321, Nov. 2000.

[9] U. Fincke and M. Pohst, "Improved method for calculating vector of short length in a lattice, including a complexity analysis," Math. Comput., vol. 44, pp. 463-471, Apr. 1985.

[10] E. Viterbo and J. Boutros, "A universal lattice code decoder for fading channels," IEEE Trans. Inf. Theory, vol. 45, no. 5, pp. 1639-1642, Jul. 1999.

[11] M. O. Damen, A. Chkeif, and J.-C. Belfiore, "Lattice code decoder for space-time codes," IEEE Commun. Lett., vol. 4, no. 5, pp. 161-163, May 2000.

[12] B. M. Hochwald and S. Ten Brink, "Achieving near-capacity on a multipleantenna channel," IEEE Trans. Commun., vol. 51, no. 3, pp. 389-399, Mar. 2003.

[13] L. Brunel, "Multiuser detection techniques using maximum likelihood sphere decoding in multicarrier CDMA systems," IEEE Trans. Wireless Commun., vol. 3, no. 3, pp. 949-957, May 2004.

[14] D. Pham, K. R. Pattipati, P. K. Willet, and J. Luo, "An improved complex sphere decoder for V-BLAST systems," IEEE Signal Process. Lett., vol. 11, no. 9, pp. 748-751, Sep. 2004.

[15] T. Cui and C. Tellambura, "Approximate ML detection for MIMO systems using multistage sphere decoding," IEEE Signal Process. Lett., vol. 12, no. 3, pp. 222-225, Mar. 2005.

[16] L. Hanzo, T. H. Liew, and B. L. Yeap, Turbo Coding, Turbo Equalisation and Space-Time Coding for Transmission Over Fading Channels. Piscataway: NJ: IEEE Press, New York: Wiley, 2002.

[17] G. Ungerböeck, "Channel coding with multilevel/phase signals," IEEE Trans. Inf. Theory, vol. IT-28, no. 1, pp. 55-67, Jan. 1982.

[18] P. Robertson and T. Wörz, "Bandwidth efficient turbo trellis-coded modulation using punctured component codes," IEEE J. Sel. Areas Commun., vol. 16, no. 2, pp. 206-218, Feb. 1998.

[19] E. Zehavi, "8-PSK trellis codes for a Rayleigh fading channel," IEEE Trans. Commun., vol. 40, no. 5, pp. 873-883, May 1992.

[20] X. Li and J. A. Ritcey, "Bit-interleaved coded modulation with iterative decoding using soft feedback," Electron. Lett., vol. 34, no. 10, pp. 942943, May 1998.

[21] M. Jiang, S. X. Ng, and L. Hanzo, "TCM, TTCM, BICM and BICM-ID assisted MMSE multi-user detected SDMA-OFDM using walsh-hadamard spreading," in Proc. IEEE Vehicular Technology Conf., Milan, Italy, 2004, pp. 1129-1133.

[22] J. Holland, Adaptation in Natural and Artificial Systems. Ann Arbor, MI: Univ. of Michigan Press, 1975.

[23] D. E. Goldberg, Genetic Algorithms in Search, Optimization, and Machine Learning. Reading, MA: Addison-Wesley, 1989.

[24] An Introduction to Genetic Algorithms. Cambridge, MA: MIT Press, 1996.

[25] M. Mitchell and D. Whitley, "A genetic algorithm tutorial," Stat. Comput., vol. 4, no. 2, pp. 65-85, Jun. 1994.

[26] S. Forrest, "Genetic algorithms: Principles of natural selection applied to computation," Science, vol. 261, no. 5123, pp. 872-878, Aug. 1993.

[27] M. J. Juntti, T. Schlösser, and J. O. Lilleberg, "Genetic algorithms for multiuser detection in synchronous CDMA," in Proc. IEEE Int. Symp. Information Theory (ISIT" 97), Ulm, Germany, 1997, p. 492. 
[28] X. F. Wang, W.-S. Lu, and A. Antoniou, "A genetic-algorithm-based multiuser detector for multiple-access communications," in Proc. 1998 IEEE Int. Symp. Circuits and Systems, vol. 4, Monterey, CA, pp. 534537.

[29] C. Ergün and K. Hacioglu, "Multiuser detection using a genetic algorithm in CDMA communications systems," IEEE Trans. Commun., vol. 48, no. 8, pp. 1374-1383, Aug. 2000.

[30] K. Yen and L. Hanzo, "Antenna-diversity-assisted genetic-algorithmbased multiuser detection schemes for synchronous CDMA systems," IEEE Trans. Commun., vol. 51, no. 3, pp. 366-370, Mar. 2003.

[31] L. Hanzo, L.-L. Yang, E.-L. Kuan, and K. Yen, Single- and Multi-Carrier DS-CDMA: Multi-User Detection, Space-Time Spreading, Synchronisation and Standards: Piscataway, NJ: IEEE Press, New York: Wiley, 2003.

[32] K. Yen and L. Hanzo, "Genetic algorithm assisted joint multiuser symbol detection and fading channel estimation for synchronous CDMA systems," IEEE J. Sel. Areas Commun., vol. 19, no. 6, pp. 985-998, Jun. 2001.

[33] S. Abedi and R. Tafazolli, "Genetically modified multiuser detection for code division multiple access systems," IEEE J. Sel. Areas Commun., vol. 20, no. 2, pp. 463-473, Feb. 2002.

[34] U. Fawer and B. Aazhang, "A multiuser receiver for code division multiple access communications over multipath channels," IEEE Trans. Commun., vol. 43, no. 2-4, pp. 1556-1565, Feb./Apr. 1995.

[35] E. Zitzler and L. Thiele, "Multiobjective evolutionary algorithms: A comparative case study and the strength pareto approach," IEEE Trans. Evol. Comput., vol. 3, no. 4, pp. 257-271, Nov. 1999.

[36] B. L. Miller and D. E. Goldberg, "Genetic algorithms, selection schemes and the varying effects of noise," Evol.Comput., vol. 4, no. 2, pp. 113-131, 1996.

[37] L. J. Eshelman and J. D. Schaffer, "Preventing premature convergence in genetic algorithms by preventing incest," in Proc. 4th Int. Conf. Genetic Algorithms, R. K. Belew and L. B. Booker, Eds., CA, 1991, pp. 115122.

[38] G. Syswerda, "Uniform crossover in genetic algorithms," in Proc. 3rd Int. Conf. Genetic Algorithms, J. D. Schaffer, Ed., CA, 1989, pp. 2-9.

[39] A. E. Eiben, R. Hinterding, and Z. Michalewicz, "Parameter control in evolutionary algorithms," IEEE Trans. Evol. Comput., vol. 3, no. 2, pp. 124 141, Jul. 1999.

[40] J. G. Proakis, Digital Communications, 4th ed. New York: McGrawHill, 2001.

[41] W. Ledermann, E. Lloyd in Handbook of Applicable Mathematics, Volume II: Probability. vol. 2, New York, Wiley, 1980.

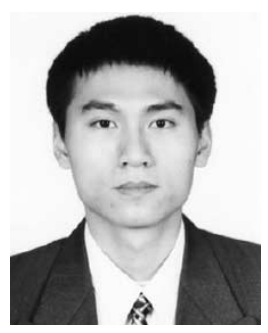

Ming Jiang (S'04) received the B.Eng. and M.Eng. degrees in electronics engineering from the South China University of Technology (SCUT), Guangzhou, China, in 1999 and 2002, respectively. $\mathrm{He}$ is currently working toward the Ph.D. degree in the Communications Group, School of Electronics and Computer Science at the University of Southampton, Southampton, U.K.

His research interests include heuristic and adaptive optimization algorithms, joint multiuser detection, space-time coding, transceiver diversity, frequency-hopping, and MIMO OFDM/MC-CDMA systems.

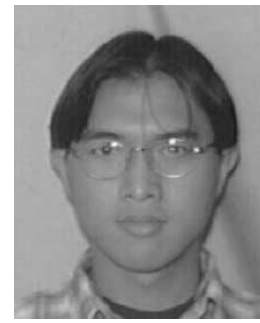

Soon Xin Ng (M'02) received the B. Eng. degree (First class) in electronics engineering and the Ph.D. degree in mobile communications from the University of Southampton, Southampton, U.K., in 1999 and 2002, respectively.

Currently, he is continuing his research as a Postdoctoral Research Fellow at the University of Southampton. His research interests are mainly in adaptive coded modulation, channel coding, turbo coding, space-time coding, and joint source and channel coding. He has published numerous papers and coauthored a book in this field.

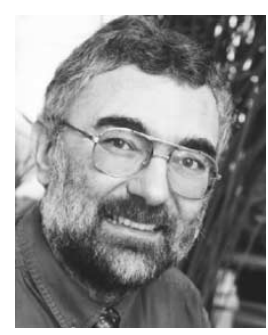

Lajos Hanzo (F'02) received the Master degree in electronics in 1976 and the Ph.D. degree in telecommunications in 1983. In 2004, he received the Doctor of Sciences degree from the University of Southampton, Southampton, U.K.

During his 28-year career in telecommunications, he has held various research and academic posts in Hungary, Germany, and the U.K. Since 1986, he has been with the Department of Electronics and Computer Science, University of Southampton, where he holds the Chair in Telecommunications. He has coauthored eleven John Wiley/IEEE Press books totalling about 9000 pages on mobile radio communications, published in excess of 500 research papers, organized and chaired conference sessions, presented overview lectures, and has been awarded numerous distinctions. Currently, he is managing an academic research team, and working on a range of research projects in the field of wireless multimedia communications sponsored by industry, the Engineering and Physical Sciences Research Council (EPSRC) U.K., the European IST Programme, and the Mobile Virtual Centre of Excellence (VCE), U.K. He is an enthusiastic supporter of industrial and academic liaison, and he offers a range of industrial courses.

Dr. Hanzo is an IEEE Distinguished Lecturer of both the Communications Society and the Vehicular Technology Society, as well as a Fellow of the IEE and the Royal Academy of Engineering (FREng.). 